\title{
Neural Activation in the Somatosensory Cortex by Electrotactile Stimulation of the Fingers: A Human fMRI Study
}

\author{
Ji-Woo Seok, Un-Jung Jang, Jin-Hun Sohn \\ Department of Psychology, Chungnam National University, Daejeon, 305-764
}

\author{
Corresponding Author \\ Jin-Hun Sohn \\ Department of Psychology, Chungnam \\ National University, Daejeon, 305-764 \\ Phone : +82-42-821-6369 \\ Email : jhsohn@cnu.ac.kr
}

Received : September 05, 2014

Revised : September 19, 2014

Accepted: September 26, 2014

Copyright@2014 by Ergonomics Society of Korea. All right reserved.

(c) This is an open-access article distributed under the terms of the Creative Commons Attribution Non-Commercial License (http:// creativecommons.org/licenses/by-nc/3.0/), which permits unrestricted non-commercial use, distribution, and reproduction in any medium, provided the original work is properly cited.
Objective: The aim of this study is to investigate 1) somatotopic arrangement of the second and third fingers in SI area 2) difference of neural activation in the SI area produced by stimulation with different frequencies 3) correlation between the intensity of tactile perception by different stimulus intensity and the level of brain activation measurable by means of fMRI.

Background: Somatosensory cortex can obtain the information of environmental stimuli about "where" (e.g., on the left palm), "what" (e.g., a book or a dog), and "how" (e.g., scrub gently or scrub roughly) to organism. However, compared to visual sense, the neural mechanism underlying the processing of specific electrotactile stimulus is still unknown.

Method: 10 right-handed subjects participated in this study. Non-painful electrotactile stimuli were delivered to two different finger tips of right hand. Functional brain images were collected from 3.0T MRI using the single-shot EPI method. The scanning parameters were as follows: TR and TE were $3000,35 \mathrm{~ms}$, respectively, flip angle 60 , FOV $24 \times 24 \mathrm{~cm}$, matrix size $64 \times 64$, slice thickness $4 \mathrm{~mm}$ (no gap). SPM5 was used to analyze the fMRI data.

Results: Significant activations produced by the stimulation were found in the $\mathrm{SI}$, SII, the subcentral gyrus, the precentral gyrus, and the insula. In all participants, statistically significant activation was observed in the contralateral SI area and the bilateral SII areas by the stimulation on the fingers but ipsilaterally dominant. The $\mathrm{SI}$ area representing the second finger generally located in the more lateral and inferior side than that of the third finger across all the subjects. But no difference in brain area was found for the stimulation of the fingers by different frequencies. And two typical patterns were observed on the relationship between the perceived psychological intensity and the amount of voxels in the primary sensory cortex during the stimulation.

Conclusion: It was possible to discriminate the representation sites in the SI by electrotactile stimulation of digit2 and digit3. But we could not find the differences of the brain areas according to different stimulation frequencies from 3 to $300 \mathrm{~Hz}$.

Application: The results of the study can provide a deeper understanding of somatosensory cortex and offer the information for tactile display for blinds.

Keywords: Electrotactile stimulation, Frequency, Functional magnetic resonance imaging, Somatosensory area 


\section{Introduction}

Tactile sensation is one of the important primary senses acquiring and judging the most basic information on object through dermal sensation. Namely, tactile sensation is a sensory function sensing physical stimulation on skin. The tactile sensation can be divided into pressure sensation, friction sensation and location sensation. A human identifies the surrounding environment and objects by accepting and integrating the sensations, and judges and evaluates the objects through emotional pleasantness and unpleasantness. Since the emotional evaluation on the objects may affect decision making on purchase, etc., it has very important significance in industrial setting. As the importance of tactile sensation emerges recently, attempts to develop technologies to perceive and embody the tactile sensation that can be utilized in robot development and clinical medicine by applying the tactile sensation increase. To develop such a tactile sensation embodying technology, it is essential to examine the mechanism of tactile sensation. Therefore, studies on nerve network and psychophusicas function on tactile sensation continue so far. Especially, as brain area activation has been effectively analyzed scientifically since 1990s, and can become visualized, due to the development of brain imaging technology (Ogawa et al., 1992), studies to examine brain mechanism are carried out, according to tactile stimulation location and physical characteristics by using brain imaging technology.

Kurth et al. (1998) proposed electro tactile stimulation on the second and fifth fingers to 20 experiment participants. As a result of individual analysis, it was identified that the activation area of the second finger was located in the more inferior and lateral side, compared to the fifth finger. Boakye et al. (2000) tried to find out the activation pattern of somatosensory area, according to frequency difference using fMRI (functional magnetic resonance imaging). $5.1 \mathrm{~Hz}$ electrotactile stimulation was presented to five participants, and $50 \mathrm{~Hz}$ electrotactile stimulation was presented to other five participants. As a result of group analysis, the SI (somatosensory cortex I) and SII (somatosensory cortex II) areas and parietal perculum, insula, frontal cortex, supplementary motor, posterior parietal cortices $(B A 7,40)$ areas were activated. According to the comparison of stimulation frequency difference, there was no difference in the $5.1 \mathrm{~Hz}$ and $50 \mathrm{~Hz}$ volumes of cortical activation in the SI and SII areas. Francis et al. (2000) presented $80 \mathrm{~Hz}$ vibrotactile stimulation to the second and fifth fingers of eight participants, and $30 \mathrm{~Hz}$ and $80 \mathrm{hz}$ vibrotactile stimulation to the second fingers of four participants. As a result, the SI and SII areas and subcentral gyrus, precentral gyrus, posterior insula, posterior parietal region, posterior cingulated areas were activated. Also, in the SI area, the cortical area of the second finger was located in the more lateral side, compared to the fifth finger. There was no significant difference in the activation area size, according to frequency. Harrington and Hunter (2001) tried to examine the somatosensory cortex differences, according to frequency, namely, the differences of Messner and Pacinian receptor in the brain cortical area. To this end, they presented non-painful stimulation to the three fingers $(1,2$ and 3 ) of seven participants using the vibrotactile buzzer. The $35 \mathrm{~Hz}$ and $150 \mathrm{~Hz}$ stimuli were presented to the three fingers for 19.2 seconds, respectively, (five times of repetition). According to SI area analysis result, BA 1, 2 and 3 were activated from three participants out of the seven participants by $35 \mathrm{~Hz}$ stimulation, and only BA 1 and 2 were activated by $150 \mathrm{~Hz}$ stimulation. According to SII area analysis result, the activated cortical area after presenting $150 \mathrm{~Hz}$ stimulation and the cortical area activated after presenting $35 \mathrm{~Hz}$ were overlapped (Harrington and Hunter, 2001).

As such, although, there were many studies to examine the brain mechanism of stimulated frequencies using $\mathrm{fMRl}$, these preceding studies mostly used only two types of frequency stimuli as the comparison of difference between low and high frequencies. And thus, there was limitation in examining brain activation mechanism. However, this study is expected to investigate influences, according to differently presented stimulation frequencies, as well as the stimulation types and variables that were not applied in the preceding studies, by making a paradigm that can diversely present frequencies ranging from low frequency to high frequency including $10 \mathrm{~Hz}, 30 \mathrm{~Hz}, 50 \mathrm{~Hz}, 150 \mathrm{~Hz}$ and $300 \mathrm{~Hz}$.

Also, this study is to verify the homunculus acquired by electrotactile stimulating patients' brains through the imaging technique. Although, the preceding studies tried to examine tactile cortical area differences responding to each finger, the existing studies tried to identify the differences in areas responding to the second and fifth fingers. This study investigates whether the classification 
of brain cortical areas responding to the second and third fingers, namely, the fingers located closely, is possible.

By analyzing the correlation of psychological data and brain cortical responses, this study intends to examine mental-physiological relationship. The studies researching the differences in activation by stimulation frequency at the initial stages mainly focused on SI area. This study also analyzes ROI (region of interest), focusing on SI area, based on the preceding studies. This study checks the response mechanism of brain cortex by tactile stimulation, and examines relations with psychological tactile sensation. Towards this end, this study is to discover functional relationship between these by identifying the dynamic characteristics of external stimuli, resultant psychological perception and relationship between responses of brain cortex using eletrotactile stimulation. In other words, this paper identifies the differences in brain activation areas, according to tactile stimulation presented in different areas. This paper investigates responses on the somatosensory cortex by the frequency of electrotactile stimulation. Lastly, this paper identifies psychological perception and relationship between somatosensory cortical responses.

\section{Method}

\subsection{Subjects}

12 undergraduate students at Chungnam National University participated in the experiment. All the participants are female and right-handed students. Their age distribution was from 19 to 25 , and they had no huge difficulties in the feeling of tactile stimulation without the history of brain injury or mental disease. The experiment participants were limited to female students in this study, and the reason is that variability related to sensitivity or the status of pain is huge, according to gender. Overall things on the experiment were explained to all the participants, and consent was acquired from them. All experiment procedures conformed to the regulations of the Institutional Review Committee.

\subsection{Electrotactile stimuli}

This study used low frequency therapeutic apparatus used in everyday life to present electrotactile stimulation. The low frequency therapeutic apparatus used in this study can present various frequencies between $3-300 \mathrm{~Hz}$, and physical intensity can be controlled from 1 to 8 . This study intends to present equal stimulation intensity, according to all frequencies, since the purpose of this study is to see the differences in brain activation responses, according to stimulation frequency. To find proper stimulation intensity, a preliminary experiment was conducted targeting 30 people. Namely, whether stimulation was felt was measured, when stimulation was presented at physical intensity of 1 to 8 , and also whether pain was felt was measured, when stimulation was presented at physical intensity of 1 to 8 . As a result, when stimulation with weak physical intensity (intensity 2 and lower) was presented at low frequency $(3 \mathrm{~Hz})$, all the participants did not perceive the stimulation, and $93.3 \%$ of the participants perceived the stimulation when stimulation with physical intensity 4 was presented. When stimulation (7 and higher) with physical intensity at $300 \mathrm{~Hz}$ was presented, all the participants felt pain and unpleasantness. When stimulation with physical intensity 4 was presented, $10 \%$ of the participants said pain was caused. When stimulation with physical intensity 5 was presented, $56.6 \%$ of the participants said pain was caused. Through this preliminary experiment, the physical intensity 4.5 to be used in this experiment was selected as proper physical intensity not causing pain, while the participants can perceive stimulation. This study composed the paradigm to present various frequencies from low to high frequencies including $3 \mathrm{~Hz}, 10 \mathrm{~Hz}, 30 \mathrm{~Hz}, 100 \mathrm{~Hz}$ and $300 \mathrm{~Hz}$ to supplement the limitation of existing preceding studies, in which frequency was limited, and to compare the differences of low and high frequencies.

Based on the preceding studies that identified the differences of brain cortex responding to the second and fifth fingers, this study presented stimulation to the second and third fingers, respectively, to find out whether brain cortical area classification responding to the fingers located closely is possible. 


\subsection{Experimental procedures and stimuli paradigm}

The experiment participants was guided to read experiment instructions and be familiar with them, as they had time to adapt to the experiment in the lab, while electrodes were attached to their fingers. To prevent noise occurrence, due to head shaking, an instruction not to move head was provided upon shooting the images. And then, about 10-min of experiment was carried out (three dummy images: nine sec, five $30 \mathrm{sec}$ blocks: five min), after lining anterior comissure and posterior commissure through about 10-min shimmering in the fMRI. After that, about 15-min of T1 image was acquired (Figure 1). The experiment stimulation paradigm consisted of two sessions with boxcar design (second and third fingers) (Figure 2), and each session was comprised of ten blocks. In each block, control stimulation and tactile stimulation $(3,10,30,100,300 \mathrm{~Hz})$ were presented alternately for $15 \mathrm{sec}$. When the experiment was finished in the $\mathrm{fMRI}$, the experiment participants evaluated psychological intensity on the stimulation of five frequencies. The experiment was conducted to each participant under the same condition each time using such a process.

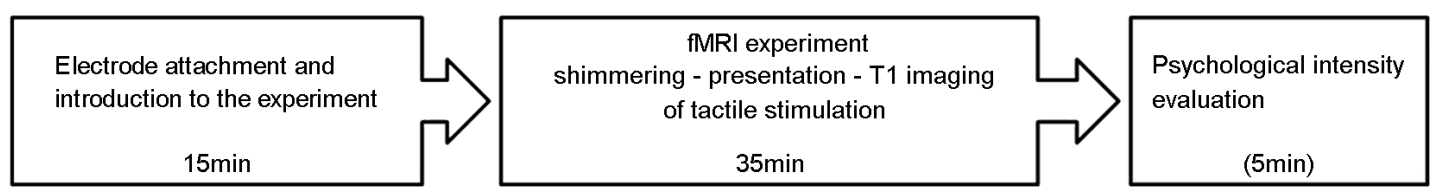

Figure 1. Experimental procedures

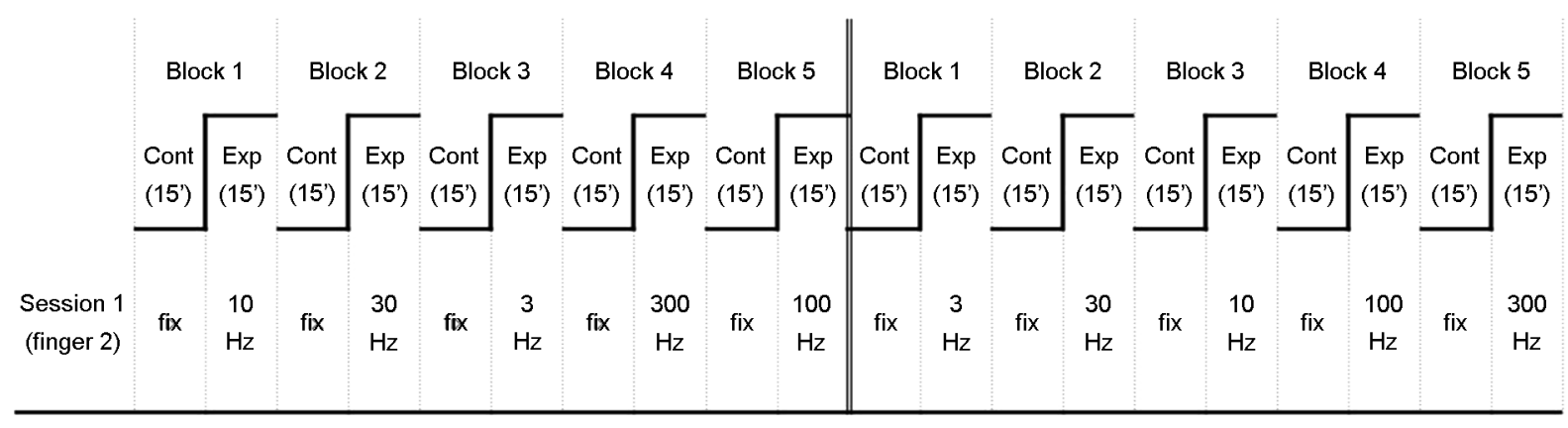

Figure 2. The example of stimuli paradigm

\subsection{Psychological data acquisition}

After the fMRI experiment was finished, the experiment participants wrote psychological evaluation scale to evaluate how much the electrotactile stimulation set was strong. To evaluate how much tactile stimulation was felt, the scale consisted of five-scale: hardly felt (one point) to very strongly felt (five points).

\subsection{MRI data acquisition and analysis}

For MRI data acquisition, ISOL 3.0T Forte installed at the fMRI Center was used. While the participants undertake the task, while feeling the tactile stimulation presented through the electrodes attached to the fingers, the MRI data was acquired using the EPI-BOLD (Echo Planner Imaging Blood Oxygen Level Dependent) technique. Other conditions were as follows: 
TR 3000, TE 35msec, slice thickness $4 \mathrm{~mm}$ no gap, 35 slices, $64 \times 64$ Matrix, FOV $24 \times 24 \mathrm{~cm}$, flip angle $60^{\circ}$. For each experiment session, 103 total volume images were acquired including three dummy images for $300 \mathrm{sec}$. Concerning anatomical brain images, T1 emphasis images were collected (TR/TE 3000/35msec, slice thickness $1 \mathrm{~mm}$ no gap, $256 \times 256$ Matrix, FOV $24 \times 24 \mathrm{~cm}$, flip angle $60^{\circ}$ ).

The fMRI data was ultimately analyzed through the following procedures using SPM 99 software (Wellcome Department of Cognitive Neurology, London, UK): slice timing indicating calibration on the image (data) acquisition time, motion-correction for head movement calibration, coregistration to coordinate anatomical images and functional images and normalization to conform individual brain to standard brain (Montreal Neurologic Institute, MNI), and then smoothing stage using 8mm Isotrophic Gaussian Kernel. From the pre-processing, two participants' brain MRI data could not be used, due to big head movement. Targeting the data from the remaining ten participants, the activation of nerve network was identified at the stimulation presentation time, when significant activation was bigger than the base condition using sustraction, after finding the statistically significantly activated brain area in each condition through the general linear model. Regarding the area coordinate confirmed in the brain function map, the brain area was checked using Talairach-Tournoux system in the MNI coordinate.

\section{Results}

\subsection{Brain activation by stimulation of fingers}

Among the 12 participants, two participants, whose head movement was severe to affect brain image signal, were excluded from brain image analysis. As a result of individual analysis, all the ten participants' contralateral SI area (brodmann area 1,2 and 3 on the left hemisphere) was activated. Among them, four participants' bilateral SI areas were activated. All the ten participants' bilateral SII areas (SII area of left and right hemispheres, brodmann area 40) were activated. As a result of group analysis, when stimulation was presented to a finger, the contralateral $\mathrm{SI}$ area was activated. The activated areas, other than the somatosensory area, were bilateral inferior frontal gyrus, bilateral middle frontal gyrus, right insula and left inferior parietal lobule (Figure 3).

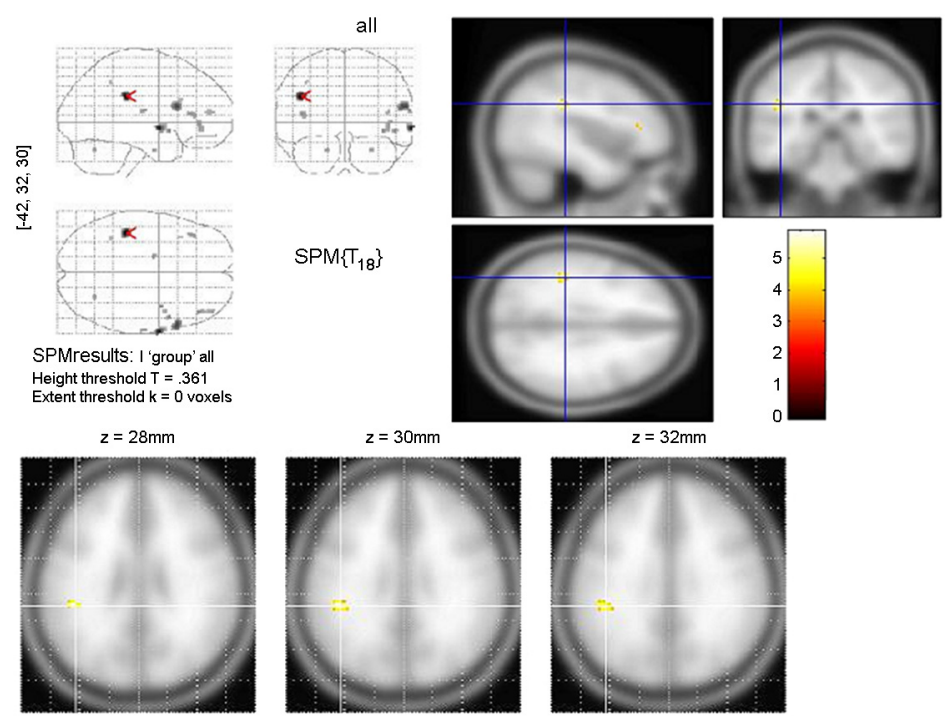

Figure 3. Brain activation by group analysis 


\subsection{Brain activation with different frequencies}

This study designated left SI area as the region of interest (ROI), and location differences were compared between activation areas, according to frequency. Through the group analysis result, Table 1 summarizes the voxel coordinates with the biggest $Z$ score, and the $\mathrm{Z}$ scores among the activated SI areas $(p<.001$, uncorrected).

Table 1. Montreal Neurological Institute (MNI) coordinates of cortical areas activated by stimuli of each frequency

\begin{tabular}{c|c|c|c|c}
\hline & \multicolumn{3}{|c|}{ Talairach coordinate } & \multirow{2}{*}{ Z-score } \\
\cline { 2 - 5 } & $\mathrm{X} \mathrm{mm}$ & $\mathrm{Y} \mathrm{mm}$ & 30 & 4.3 \\
\hline 3 & -40 & -32 & 34 & 4.5 \\
\hline 10 & -48 & -22 & 56 & 3.93 \\
\hline 30 & -32 & -42 & 44 & 5.89 \\
\hline 100 & -50 & -34 & 52 & 4.5 \\
\hline
\end{tabular}

The following figure shows $\mathrm{SI}$ area that is activated in the contrast condition of each stimulation presentation and base state (Figure 4). As a result of group analysis, there was difference in voxel location having the biggest $Z$ score within the activated area, according to frequency; however, there were many parts, where activation cluster was overlapped overall.

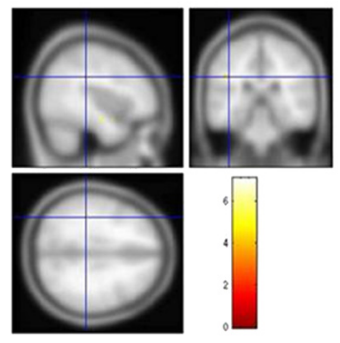

$3 \mathrm{~Hz}$

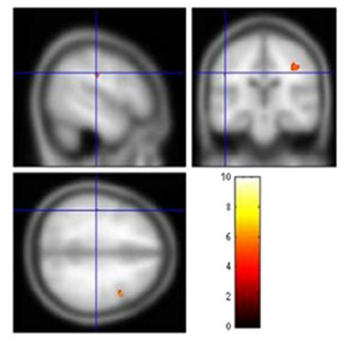

$10 \mathrm{~Hz}$

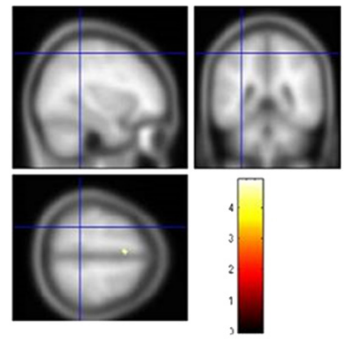

$30 \mathrm{~Hz}$

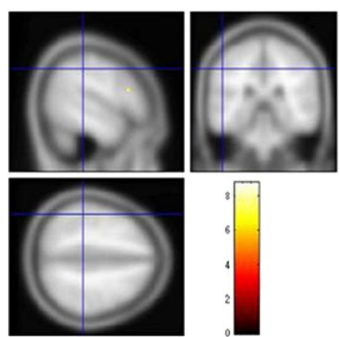

$100 \mathrm{~Hz}$

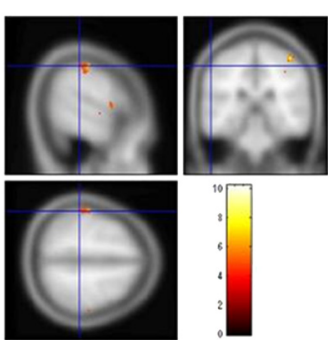

$300 \mathrm{~Hz}$

Figure 4. Brain activations with different frequencies $(3 \mathrm{~Hz}, 10 \mathrm{~Hz}, 30 \mathrm{~Hz}, 100 \mathrm{~Hz}, 300 \mathrm{~Hz})$ 


\subsection{Brain activation area by stimulation of two different fingers}

From all the ten participants, the locations of brain area responding to the second and third fingers were differently demonstrated, respectively (Table 2). The following figure shows activation within the SI area of a participant (Figure 5). From seven out of the ten participants, the somatosensory cortex area representing the second finger was located in the more lateral and inferior side than the somatosensory cortex area representing the third finger.

Table 2. Montreal Neurological Institute (MNI) coordinates of different finger representation

\begin{tabular}{c|c|c|c|c|c|c}
\hline \multirow{2}{*}{ Participant } & \multicolumn{3}{|c}{ Second finger } & \multicolumn{3}{c}{ Third finger } \\
\cline { 2 - 7 } & $\mathrm{X} \mathrm{mm}$ & $\mathrm{Y} \mathrm{mm}$ & $\mathrm{Z} \mathrm{mm}$ & $\mathrm{X} \mathrm{mm}$ & $\mathrm{Y} \mathrm{mm}$ & $\mathrm{Zmm}$ \\
\hline 1 & -55.3 & -35.5 & 52.8 & -19.4 & -31.7 & 53.6 \\
\hline 2 & -63.6 & -27.9 & 45.5 & -53.7 & -27.9 & 47.2 \\
\hline 3 & -43.1 & -31.7 & 52.8 & -40.0 & -30.1 & 58.2 \\
\hline 4 & -18.7 & -53.4 & 56.7 & -49.9 & -52.9 & 50.6 \\
\hline 5 & -37.7 & -21.4 & 55.1 & -65.9 & -33.6 & 43.7 \\
\hline 6 & -49.9 & -13.4 & 49 & -49.2 & -15.7 & 56.7 \\
\hline 7 & -47.6 & -36.7 & 48.3 & -35.4 & -51.9 & 53.6 \\
\hline 8 & -31.7 & -39.4 & 50.0 & -35.5 & -45.0 & 51.9 \\
\hline 9 & -58.3 & -23.7 & 46.0 & -37.7 & -31.7 & 45.2 \\
\hline 10 & -56.8 & -23.7 & 47.4 & -45.3 & -26.3 & 49.0 \\
\hline
\end{tabular}
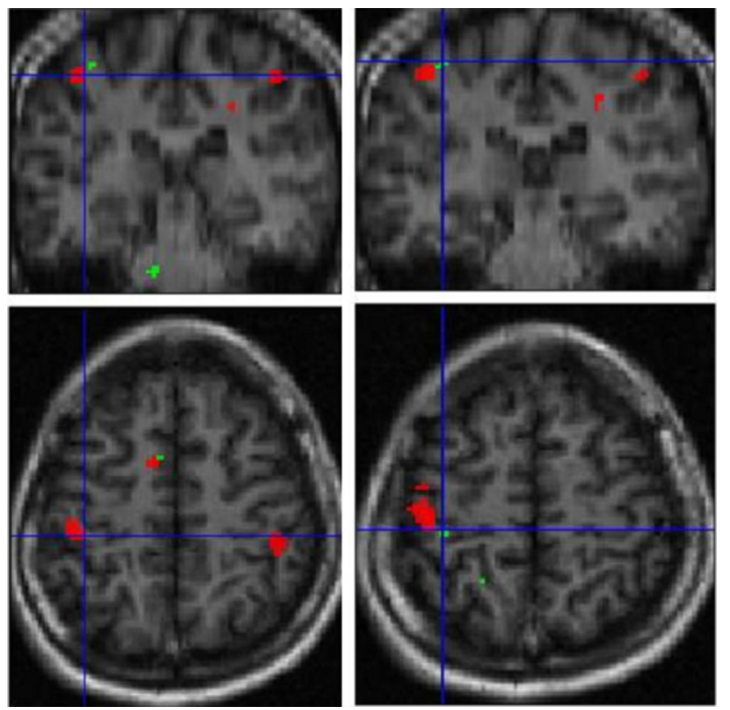

Figure 5. The example of brain activation by stimulation of two different fingers (Red: second finger, Green: third finger) 


\subsection{Voxel size by stimulation of different frequencies}

To check the brain area size activated according to different frequencies, this study calculated the number of statistically significantly activated voxels within the SI area. Figure 6 shows the response pattern on the number of voxels activated, according to frequency by individual. As the frequency increased, the response pattern of the number of activated voxels was not demonstrated in a consistent form, and differences between individuals were huge.

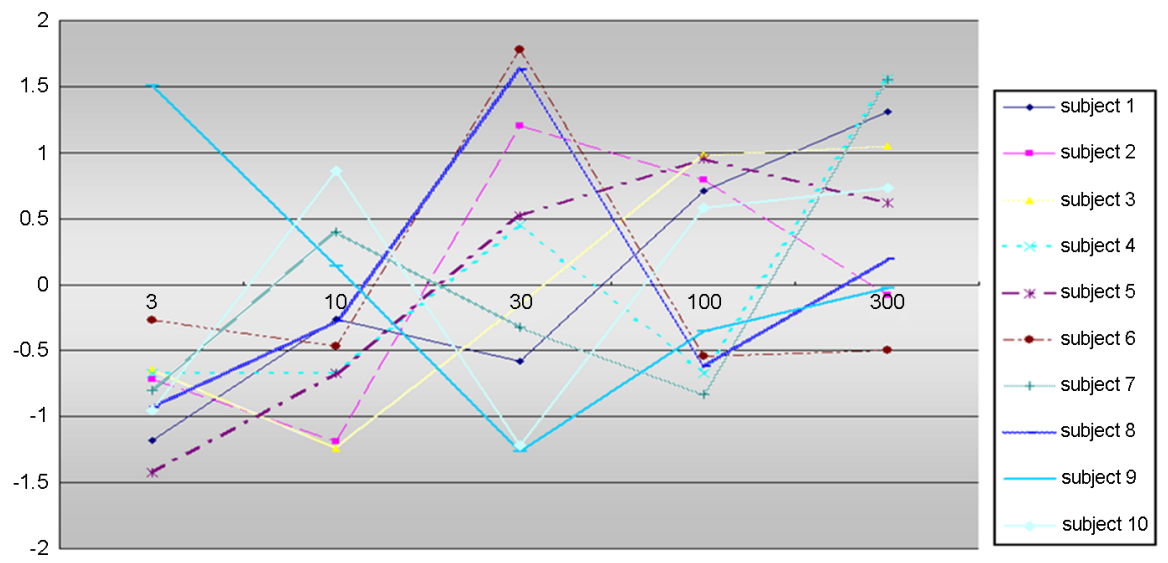

Figure 6. Activation pattern in the SI area by different frequencies by individuals ( $\mathrm{x}$ axis: frequence, y axis: number of activated voxels)

\subsection{The relationship between the perceived intensity and brain activation}

Each individual's psychological evaluation intensity by frequency and the response pattern of activated voxels took similar forms (Figure 7). Typical two pattern relationship was observed between the perceived psychological intensity and the number of activated voxels in SI, while stimulation was presented (Figure 7). Very interestingly, as the perceived intensity on the stimulation
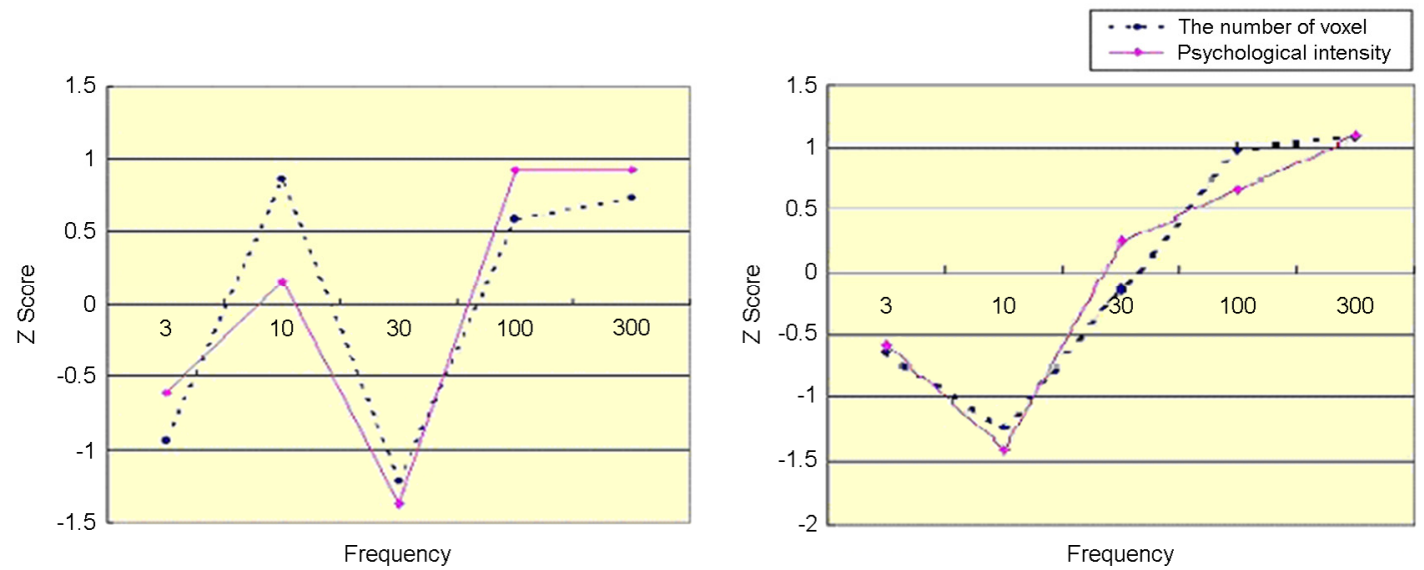

Figure 7. Two typical patterns for the relationship between the perceived intensity and brain activation ( $x$ axis represents stimulation frequencies, exponentially described) 
was stronger, the activation of SI area increased, irrelevant of pattern, and a strong positive correlation was confirmed $(t=2.58$, $p<0.05, r=0.71)$.

\section{Conclusion}

The group analysis result is consistent with other imaging study results that electrotactile stimulation activates the contralateral SI area and bilateral SII areas (Boakye et al., 2000; Francis et al., 2000; Kurth et al., 1988). The activated areas other than the somatosensory area are Bilateral Inferior Frontal Gyrus, Bilateral Middle Frontal Gyrus, Right Insula and Left Inferior Parietal Lobule, which are almost consistent with the recent preceding study result using the neuroimaging technique (Burton et al., 1993; Disbrow et al., 2000; Gelnar et al., 1998; Ledburg et al., 1995; Polonara et al., 1999; Reed et al., 1998; Ruben et al., 2001). As a result of group analysis on the differences in brain's activated locations, according to frequency, by designating $\mathrm{SI}$ area as $\mathrm{ROI}$ area, there were many overlapped parts in the activated cluster, and location differences were not consistent, according to individual. This is partially consistent with the research result of Boakye et al. (2000), and the frequency size and activated location are predicted to have no relevance.

The result in this study demonstrates the somatosensory cortical area representing to the second finger is located in the more lateral and inferior side than the somatosensory cortical area representing the third finger. This finding is consistent with the homunculus made by electrotactile stimulating the brain of brain tumor patients, and also is similar to the study result of Francis et al. (2000) that examined the location differences of brain cortical area responding to the second and fifth fingers through fMRI.

To find out brain cortex activation, when different frequencies were presented, the brain response demonstrated according to change in stimulation's physical intensity was measured, while the participants received stimulation passively. The activation response pattern, according to frequency presented to each individual, did not show consistent result and individual differences were huge.

Assuming that individual's perceived psychological factors hugely affect the activation of somatosensory cortical area, rather than by stimulation's physical characteristics, this study verified the response patterns of psychological evaluation intensity and the number of activated voxels. As a result, similar psychological pattern and brain activation pattern within each participant were exhibited, although, psychological response pattern or the response pattern of central nervous system was not consistent between each experiment participant. This implies that brain activation is more related with individual's perceived psychological cognitive factors than stimulation's physical characteristics.

The limitation of this study is that the number of experiment participants was so small. Although, the location classification of somatosensory area representing the second and third fingers was possible, according to the study result, there was a difficulty in generalizing the result acquired from the ten participants. In the further study, it is necessary to generalize the study result by increasing the number of cases. Consistent central nervous system response pattern was not shown by each individual, and there was big difference in the number of activated voxels within one individual. The reason seems that signal and noise were not discerned well, due to unstable state of the fMRI equipment used in this study. For this reason, it is necessary to ensure stability in generalizing response pattern by identifying the consistency of central nervous system response, according to physical and psychological intensities demonstrated within each individual in the further study. In addition, the preceding studies of Francis et al. (2000) and Harrington \& Hunter (2001) developed electrotactile stimulation devices and used them, however, this study used low frequency therapeutic apparatus and different stimuli were presented according to frequency, instead of using an elecrotactile stimulation device as shown in the preceding studies, since the electrotactile stimulation device could not be used in this study. Consequently, the physical characteristics-force $(\mathrm{N})$ and presented area size $(\mathrm{cm} 2)$ of the presented tactile stimulation were not 
controlled. For further study, after the experiment in this study, the development of an electrotactile stimulation device as shown in the preceding studies seems to be necessary.

Despite such a limitation, this study examined the brain cortical activation mechanism according to the presented location and size of tactile stimulation, and identified that the brain activation response of tactile stimulation is correlated with psychological and cognitive factors, rather than the presented physical size. The basic study on the examination of brain nerve network of tactile sensation is expected to be used in the tactile perception, embodiment and artificial neuroinformatics technology fields.

\section{Acknowledgements}

This work was funded by grants from Korean Federation of Science and Technology Societies (No. NRF-2006-2005087).

\section{References}

Boakye, M., Huckins, S.C., Szeverenyi, N.M., Taskey, B.I. and Hodge Jr, C.J., Functional magnetic resonance imaging of somatosensory cortex activity produced by electrical stimulation of the median nerve or tactile stimulation of the index finger, Journal of neurosurgery, 93(5), 774-783, 2000.

Burton, H., Videen, T. and Raichle, M., Tactile-vibration-activated foci in insular and parietal-opercular cortex studied with positron emission tomography: mapping the second somatosensory area in humans, Somatosensory \& motor research, 10(3), 297-308, 1993.

Disbrow, E., Roberts, T., Poeppel, D. and Krubitzer, L., Evidence for interhemispheric processing of inputs from the hands in human S2 and PV, Journal of Neurophysiology, 85(5), 2236-2244, 2001.

Francis, S., Kelly, E., Bowtell, R., Dunseath, W., Folger, S. and McGlone, F., fMRI of the responses to vibratory stimulation of digit tips, Neuroimage, 11(3), 188-202, 2000.

Gelnar, P.A., Krauss, B.R., Szeverenyi, N.M. and Apkarian, A.V., Fingertip representation in the human somatosensory cortex: an fMRI study, Neuroimage, 7(4), 261-283, 1998.

Harrington, G.S. and Downs III, H., FMRI mapping of the somatosensory cortex with vibratory stimuli: Is there a dependency on stimulus frequency?, Brain research, 897(1), 188-192, 2001.

Kurth, R., Villringer, K., Mackert, B.M., Schwiemann, J., Braun, J., Curio, G., Villringer, A. and Wolf, K.J., fMRI assessment of somatotopy in human Brodmann area 3b by electrical finger stimulation, Neuroreport, 9(2), 207-209, 1998.

Ledberg, A., O'Sullivan, B.T., Kinomura, S. and Roland, P.E., Somatosensory activations of the parietal operculum of man. A PET study, European Journal of Neuroscience, 7(9), 1934-1941, 1995.

Ogawa, S., Tank, D.W., Menon, R., Ellermann, J.M., Kim, S.G., Merkle, H. and Ugurbil, K., Intrinsic signal changes accompanying sensory stimulation: functional brain mapping with magnetic resonance imaging, Proceedings of the National Academy of Sciences, 89(13), 5951-5955, 1992.

Polonara, G., Fabri, M., Manzoni, T. and Salvolini, U., Localization of the first and second somatosensory areas in the human 
cerebral cortex with functional MR imaging, American Journal of Neuroradiology, 20(2), 199-205, 1999.

Reed, C.L., Shoham, S. and Halgren, E., Neural substrates of tactile object recognition: an fMRI study, Human brain mapping, $21(4), 236-246,2004$.

Ruben, J., Schwiemann, J., Deuchert, M., Meyer, R., Krause, T., Curio, G., Villringer, K., Kurth, R. and Villringer, A., Somatotopic organization of human secondary somatosensory cortex, Cerebral Cortex, 11(5), 463-473, 2001.

\section{Author listings}

Ji-Woo Seok: suk6124@naver.com

Highest degree: M.A.

Position title: Ph.D. candidate

Areas of interest: Neuroscience, Neuroimaging, Addiction, Psychiatry

Un-Jung Jang: zlz|852123@naver.com

Highest degree: B.A.

Position title: M.A. candidate

Areas of interest: Neuroscience, Neuroimaging, Addiction, Psychiatry

Jin-Hun Sohn: jhsohn@cnu.ac.kr

Highest degree: Ph.D.

Position title: Professor

Areas of interest: Neuroscience, Neuroimaging, Emotion, Addiction, Psychiatry 